\title{
Article \\ Energy Metabolites as Biomarkers in Ischemic and Dilated Cardiomyopathy
}

\author{
Jan Haas ${ }^{1,2}$, Karen S. Frese ${ }^{1,2}$, Farbod Sedaghat-Hamedani 1,2 (D), Elham Kayvanpour 1,2, Rewati Tappu 1,2 (D), \\ Rouven Nietsch ${ }^{1}$, Oguz Firat Tugrul 1,2, Michael Wisdom 1,2, Carsten Dietrich ${ }^{3}$, Ali Amr 1,2, Tanja Weis 1,2, \\ Torsten Niederdränk ${ }^{3}{ }^{(D}$, Michael P. Murphy ${ }^{4}$, Thomas Krieg ${ }^{5} \mathbb{D}$, Marcus Dörr 2,6 ${ }^{(D)}$, Uwe Völker 2,7 (D), \\ Jens Fielitz ${ }^{2,6}$, Norbert Frey ${ }^{1,2}$, Stephan B. Felix ${ }^{2,6}$, Andreas Keller ${ }^{8}$, Hugo A. Katus ${ }^{1,2}$ and \\ Benjamin Meder 1,2,9,*
}

check for updates

Citation: Haas, J.; Frese, K.S.; Sedaghat-Hamedani, F.; Kayvanpour, E.; Tappu, R.; Nietsch, R.; Tugrul, O.F. Wisdom, M.; Dietrich, C.; Amr, A.; et al. Energy Metabolites as Biomarkers in Ischemic and Dilated Cardiomyopathy. Int. J. Mol. Sci. 2021, 22, 1999. https://doi.org/10.3390/ ijms22041999

Academic Editor: Cristi L. Galindov Received: 15 January 2021

Accepted: 9 February 2021

Published: 18 February 2021

Publisher's Note: MDPI stays neutral with regard to jurisdictional claims in published maps and institutional affiliations.

Copyright: (c) 2021 by the authors. Licensee MDPI, Basel, Switzerland. This article is an open access article distributed under the terms and conditions of the Creative Commons Attribution (CC BY) license (https:/ / creativecommons.org/licenses/by/ $4.0 /)$.
1 Department of Internal Medicine III, University of Heidelberg, 69120 Heidelberg, Germany; jan.haas@med.uni-heidelberg.de (J.H.); karen.frese@med.uni-heidelberg.de (K.S.F.); Farbod.Sedaghat-Hamedani@med.uni-heidelberg.de (F.S.-H.);

Elham.Kayvanpour@med.uni-heidelberg.de (E.K.); Rewati.Tappu@med.uni-heidelberg.de (R.T.); Rouven.Nietsch@med.uni-heidelberg.de (R.N.); OguzFirat.Tugrul@med.uni-heidelberg.de (O.F.T.); michaelwisdom03@gmail.com (M.W.); Ali.Amr@med.uni-heidelberg.de (A.A.);

Tanja.Weis@med.uni-heidelberg.de (T.W.); Norbert.Frey@med.uni-heidelberg.de (N.F.); Hugo.Katus@med.uni-heidelberg.de (H.A.K.)

2 DZHK (German Centre for Cardiovascular Research), 17475 Greifswald, Germany; mdoerr@uni-greifswald.de (M.D.); voelker@uni-greifswald.de (U.V.); jens.fielitz@uni-greifswald.de (J.F.); Stephan.Felix@med.uni-greifswald.de (S.B.F.)

3 Siemens Healthcare GmbH, 91058 Erlangen, Germany; dietrich.carsten@siemens-healthineers.com (C.D.); torsten.niederdraenk@siemens-healthineers.com (T.N.)

4 MRC Mitochondrial Biology Unit, University of Cambridge, Cambridge CB2 0XY, UK; mpm@mrc-mbu.cam.ac.uk

5 Department of Medicine, University of Cambridge, Addenbrooke's Hospital, Hills Road, Cambridge CB2 0QQ, UK; tk382@medschl.cam.ac.uk

6 Department of Internal Medicine B, University Medicine Greifswald, 17475 Greifswald, Germany

7 Department of Functional Genomics, Interfaculty Institute for Genetics and Functional Genomics, University Medicine Greifswald, 17475 Greifswald, Germany

8 Department of Bioinformatics, University of Saarland, 66123 Saarbrücken, Germany; andreas.keller@ccb.uni-saarland.de

9 Genome Technology Center, Stanford University, Stanford, CA 94304, USA

* Correspondence: benjamin.meder@med.uni-heidelberg.de; Tel.: +49-(0)-6221-5639564; Fax: +49-(0)-6221-564645

Abstract: With more than 25 million people affected, heart failure (HF) is a global threat. As energy production pathways are known to play a pivotal role in $\mathrm{HF}$, we sought here to identify key metabolic changes in ischemic- and non-ischemic HF by using a multi-OMICS approach. Serum metabolites and mRNAseq and epigenetic DNA methylation profiles were analyzed from blood and left ventricular heart biopsy specimens of the same individuals. In total we collected serum from $n=82$ patients with Dilated Cardiomyopathy (DCM) and $n=51$ controls in the screening stage. We identified several metabolites involved in glycolysis and citric acid cycle to be elevated up to 5.7-fold in DCM $\left(p=1.7 \times 10^{-6}\right)$. Interestingly, cardiac mRNA and epigenetic changes of genes encoding rate-limiting enzymes of these pathways could also be found and validated in our second stage of metabolite assessment in $n=52 \mathrm{DCM}, n=39$ ischemic HF and $n=57$ controls. In conclusion, we identified a new set of metabolomic biomarkers for HF. We were able to identify underlying biological cascades that potentially represent suitable intervention targets.

Keywords: cardiomyopathy; energy metabolism; heart failure; multi-omics 


\section{Introduction}

Heart failure (HF) is considered as a global pandemic, since it affects around 25 million people worldwide [1,2]. It is a complex syndrome with reduced ability of the heart to pump sufficient volumes of blood [3-5] required to meet the metabolic demands of our organ systems [4]. The concept, that heart failure is comparable to an "engine out of fuel" has been developed and decades of studies on myocardial energetics have confirmed and refined this concept [6]. Briefly, the cardiac energy metabolism has three pillars: (i) uptake and utilization of fatty acids and glucose by beta-oxidation and glycolysis, respectively, (ii) oxidative phosphorylation in mitochondria, to generate energy and (iii) energy transfer and consumption [6]. The healthy heart is a "metabolic omnivore" with flexibility in substrate consumption. Whereas under normal conditions $\sim 60-90 \%$ of ATP is produced via fatty acid (FA) oxidation and only $\sim 10-40 \%$ of the ATP results from pyruvate oxidation, this balance can dramatically shift [1]. Under exercise conditions, less FA but glucose is used for ATP generation. Furthermore, stressors like prolonged fasting or poorly controlled diabetes can lead to a major shift in energy production through breakdown of ketone bodies and amino acids [1]. The numerous studies that underlie our knowledge on HF-related metabolic changes used mainly mouse models [1,2].

Human clinical studies on the metabolic changes in HF are sparse. The rationale of this study was to establish key metabolic changes during HF and find common markers of the two major forms of systolic HF, namely ischemic- and non-ischemic HF. Here, we show that several energy metabolism-related markers in the serum and the mRNA levels of their rate-limiting enzymes in the heart and whole blood are altered in patients with chronic HF, rendering them diagnostic and potential therapeutic targets.

\section{Results}

\subsection{Detection of Dysregulated Energy Metabolites}

Our study used an initial stage with a multi-omics design in non-ischemic HF and a succeeding independent validation in non-ischemic as well as ischemic HF. We collected serum from $n=82 \mathrm{HF}$ patients with DCM (mean NYHA class 2.2) and $n=51$ clinical controls without signs or symptoms of HF (NYHA class 0 ) in the first stage and from $n=52$ DCM, $n=39 \mathrm{ICM}$ and $n=57$ controls in the second stage. Clinical baseline characteristics for the subjects are summarized in Tables 1 and 2.

Table 1. Patient characteristics of the stage 1 cohorts.

\begin{tabular}{ccc}
\hline Characteristics & DCM $(\boldsymbol{n}=\mathbf{8 2})$ & Control $(\boldsymbol{n}=\mathbf{5 1})$ \\
\hline Male gender, $\mathrm{n}(\%)$ & $67(82)$ & $40(78)$ \\
Age at visit [years] $( \pm \mathrm{SD})$ & $53.52( \pm 13.53)$ & $56.22( \pm 8.75)$ \\
BMI [kg/m $\left./ \mathrm{m}^{2}\right]( \pm \mathrm{SD})$ & $28.34( \pm 6.14)$ & $25.64( \pm 2.89)$ \\
NYHA [I-IV] $( \pm \mathrm{SD})$ & $2.15( \pm 0.78)$ & 0 \\
LVEF (echo) [\%] $( \pm \mathrm{SD})$ & $31.05( \pm 13.60)$ & $61.67( \pm 3.61)$ \\
eGFR [mL/min/1.73qm BSA] $( \pm \mathrm{SD})$ & $87.48( \pm 21.62)$ & $90.54( \pm 11.08)$ \\
Smoking & & $1(2)$ \\
yes n $(\%)$ & $18(22)$ & $46(90)$ \\
no n $(\%)$ & $41(20)$ & $4(8)$ \\
ex n $(\%)$ & $22(27)$ & $0(0)$ \\
Diabetes & $12(15)$ & \\
\hline
\end{tabular}

SD, standard deviation; BMI, body mass index; NYHA, New York Heart Association; LVEF, left ventricular ejection fraction; eGFR, estimated glomerular filtration rate.

Metabolomic measurements in the stage 1 cohorts were performed using Biocrates Metabolite assays for most important energy metabolites. While some metabolites showed unaltered levels in DCM compared to controls, such as pyruvic acid (+oxaloacetic acid) (Figure 1), a range of other energy metabolites were significantly changed in their serum levels. As shown by principal component analysis, cases and controls separate very well based on their metabolic profile (Supplemental Figure S2). In case of lactate, DCM 
patients showed as much as a 5.7-fold higher level compared to controls $\left(p=1.7 \times 10^{-6}\right)$ (Figure 2). Also, Alpha-ketoglutaric acid, ( $p=4.1 \times 10^{-5} ; 1.9$-fold) (Figure 3 ) and succinic acid $\left(p=7.7 \times 10^{-7} ; 1.4\right.$-fold) (Figure 4) showed significantly increased levels in DCM patients. Together, this data points towards a changed energy metabolism in non-ischemic HF.

Table 2. Patient characteristics of the stage 2 cohorts.

\begin{tabular}{cccc}
\hline Characteristics & DCM $(\boldsymbol{n}=\mathbf{5 2})$ & ICM $(\boldsymbol{n}=\mathbf{3 9 )}$ & Control $(\boldsymbol{n}=\mathbf{5 7})$ \\
\hline Male gender, $\mathrm{n}(\%)$ & $34(67)$ & $32(82)$ & $33(58)$ \\
Age at visit [years] $( \pm \mathrm{SD})$ & $62.12( \pm 12.94)$ & $68.08( \pm 11.90)$ & $62.37(11.47)$ \\
LVEF (echo) $[\%]( \pm \mathrm{SD})$ & $28.03( \pm 11.91)$ & $37.70( \pm 21.06)$ & $58.25(24.27)$ \\
Smoking & & & \\
yes n $(\%)$ & $5(11)$ & $8(21)$ & $19(11)$ \\
no n $(\%)$ & $31(66)$ & $17(44)$ & $13(23)$ \\
ex n $(\%)$ & $11(23)$ & $14(36)$ & $33(58)$ \\
Diabetes & $11(23)$ & $13(33)$ & $3(5)$ \\
\hline
\end{tabular}

SD, standard deviation; LVEF, left ventricular ejection fraction.

2.2. Myocardial Epigenetic Programs and mRNA Levels of Rate-Limiting Enzymes Are Associated with HF Metabolites

To explore whether the metabolic state-change is due to expression changes in key enzymes of the detected metabolites, we compared the metabolite data to whole-transcriptome RNA-sequencing data from blood and heart muscle tissue from the same cohort of DCM patients and compared them to controls.

As proxies for the metabolites we chose the rate-limiting enzymes hexokinase 1 (HK1), muscle phosphofructokinase (PFKM), phosphoglycerate kinase 1 (PGK1) and muscle pyruvate kinase (PKM) for pyruvic acid (glycolysis). As part of the citric acid cycle we used oxoglutarate dehydrogenase (OGDH) and dihydrolipoamide s-succinyltransferase (DLST) for alpha-ketoglutaric acid, succinate-coA ligase alpha subunit (SUCLG1), succinate-coA ligase GDP-forming beta subunit (SUCLG2) and succinate-coA ligase ADP-forming beta subunit (SUCLA2) for succinic acid.

As shown in Figure 1, we found a significant increase in PKM (blood and heart) and a decrease in PGK1 expression (heart) in DCM patients. We also observed a significantly increased HK1 (blood and heart) (Supplemental Figure S3) and decreased LDHA (blood and heart) (Figure 2) expression. Furthermore, a significant regulation was detected for DLST (blood), OGDH (blood and heart) and ACO1 (blood and heart) expression (Figure 3). Finally, we found a decreased expression for SUCLA2 and SUCLG2 (both blood and heart) and for SUCLG1 (blood) (Figure 4). Supplemental Figure S4 is giving a comprehensive overview on the described gene expression and its validation. Of note, the corresponding methylation sites were also significantly different for those genes (Supplemental Figure S5).

\subsection{Validation of Metabolic Dysregulation for the Development of New Biomarkers}

To substantiate these initial findings on energy metabolic state-changes and to screen for robust data for the development of new biomarkers for DCM and systolic HF in general, we assessed the metabolites of the stage 1 cohorts and additional energy metabolites in independent HF cohorts (DCM, ICM, controls).

As shown in Figure 5, alpha-ketoglutaric acid and succinic acid (see Figure 5) were significantly increased in DCM and ICM as observed in the stage 1 cohorts. 


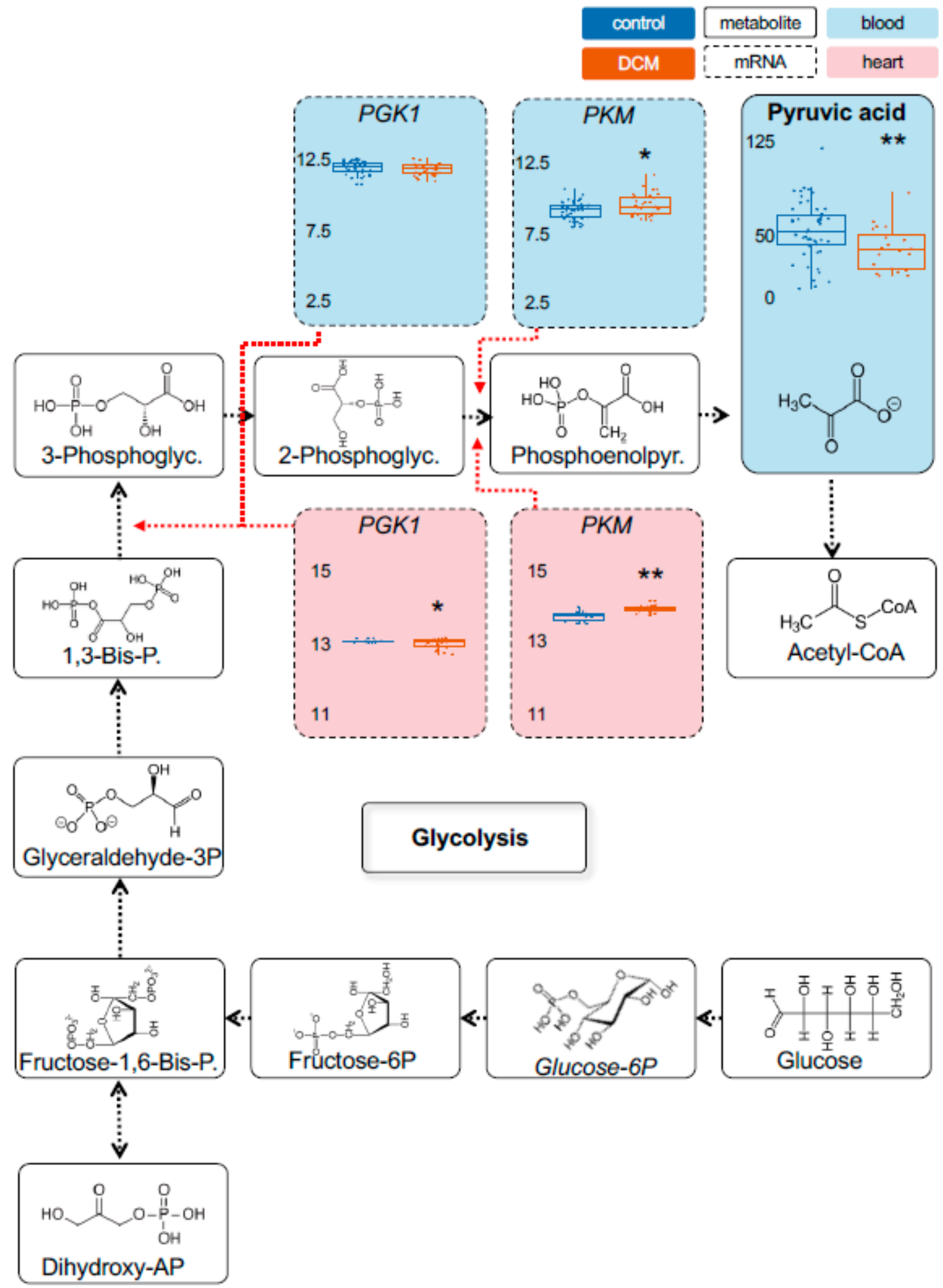

Figure 1. Detection of metabolic changes in glycolysis in heart failure (HF) patients. Control (CTRL) samples are depicted in blue. Values of the Dilated Cardiomyopathy (DCM) cohort are colored orange. Significance values are indicated. Boxes with dashed lines indicate mRNA expression and with full lines metabolite levels in serum (light blue shading) and left-ventricular heart tissue (pale red shading). As shown, PKM expression is upregulated in blood cells and myocardial tissue, while the resulting metabolite pyruvic acid was not significantly altered. Metabolite concentrations are shown as $[\mu \mathrm{M}]$. RNAseq expression is given as (rlog-)normalized read counts. ${ }^{*}: p<0.05 ;{ }^{* *}: p<0.01$. PGK1 = phosphoglycerate kinase 1 ; PKM = pyruvate kinase. 


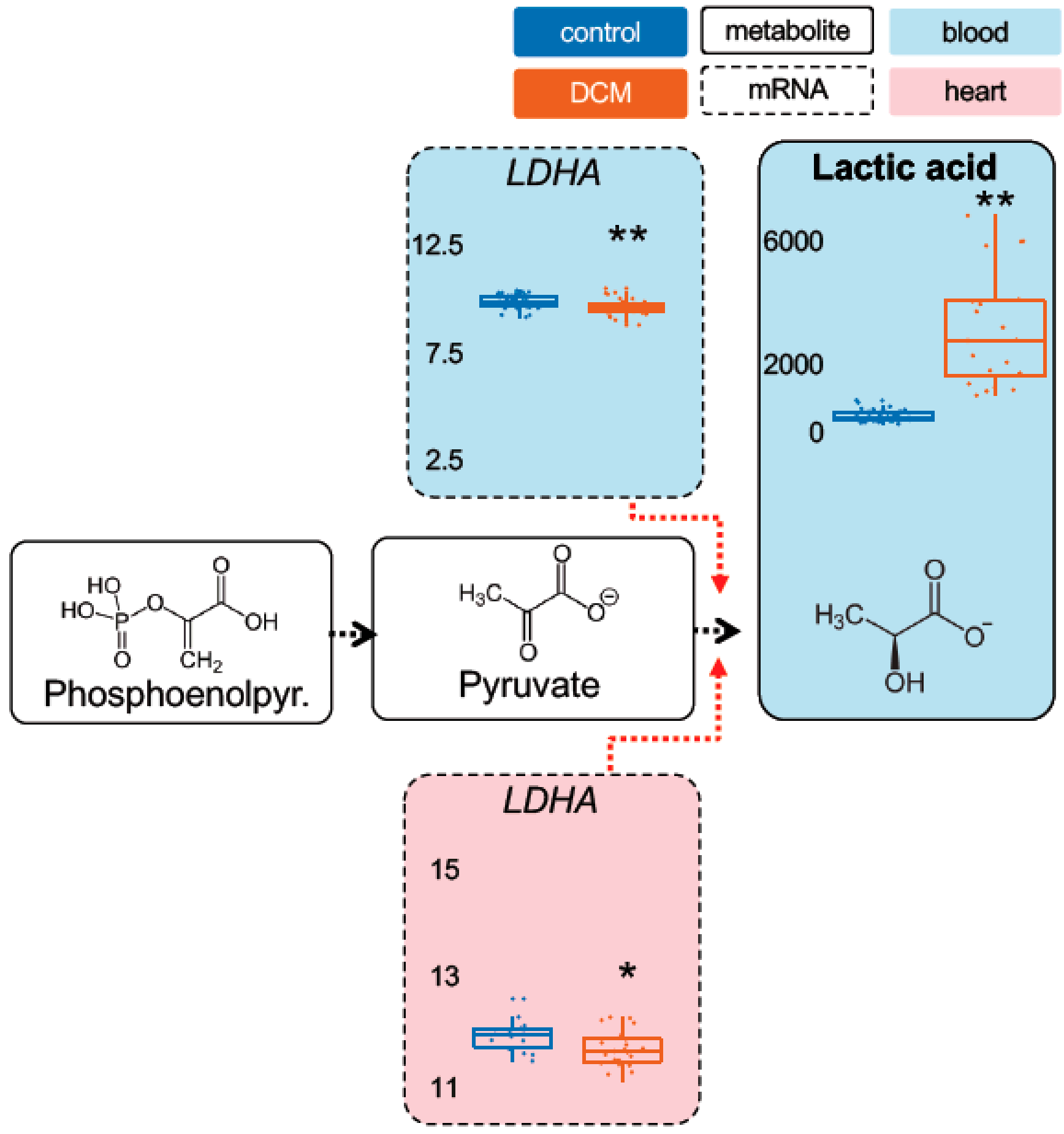

Figure 2. Detection of increased lactate levels. Following glycolysis, lactic acid is significantly increased in the stage 1 cohort, coinciding with decreased LDHA expression in blood but not in myocardial tissue. Same color and symbol annotation as in Figure 1. RNAseq expression is given as (rlog-)normalized read counts. Metabolite concentrations are shown as $[\mu \mathrm{M}]$. ${ }^{*}: p<$ $0.05 ; * *: p<0.01$. LDHA $=$ lactate dehydrogenase A. 


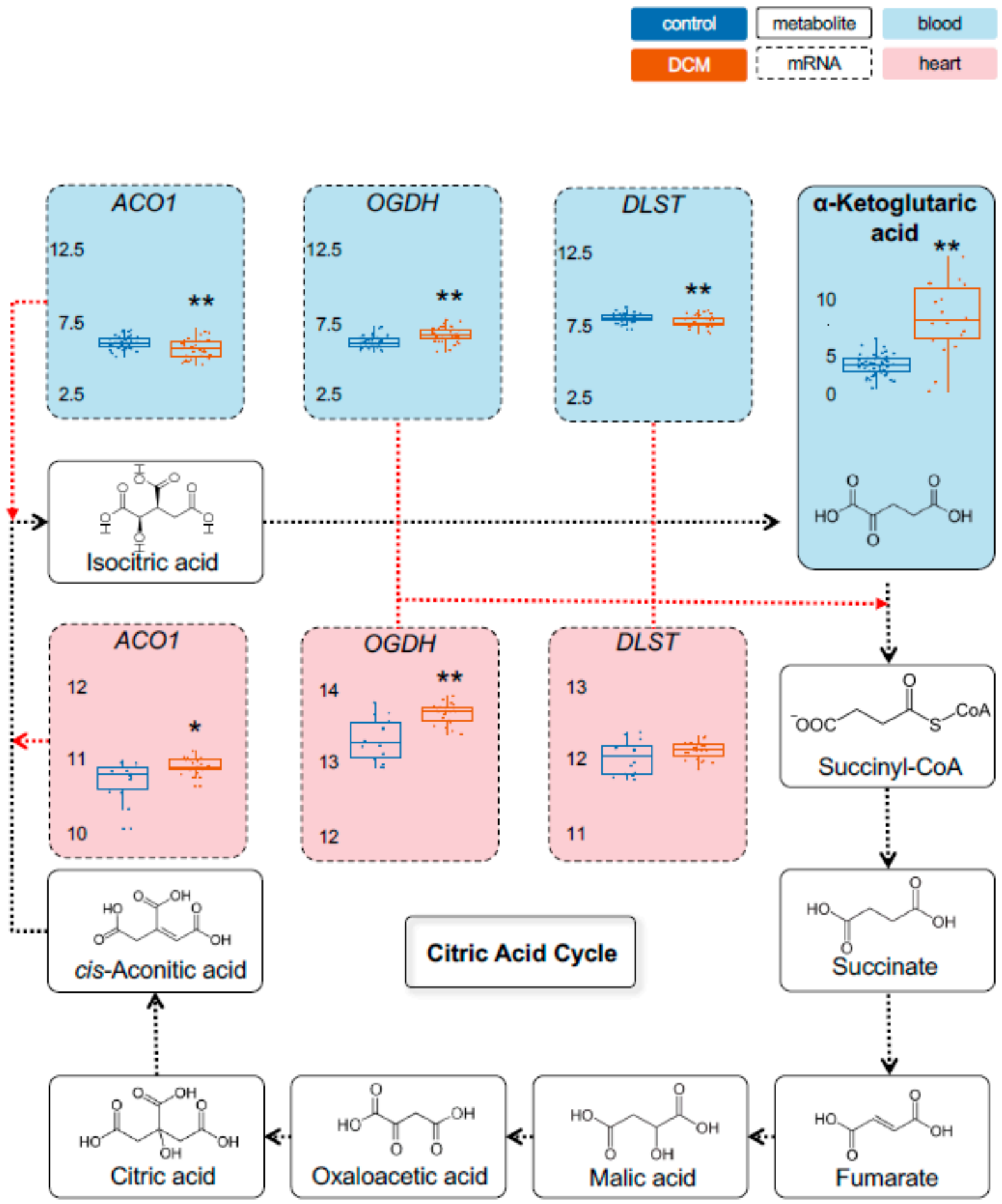

Figure 3. Detection of metabolic dysregulation in citric acid cycle. In the citric acid cycle, several mRNA expression changes were observed, with OGDH being upregulated in mononuclear blood cells and myocardial tissue. The metabolite $\alpha$-Ketoglutaric acid was (as a consequence) strongly increased and could potentially serve as new biomarker in HF. Control (CTRL) samples are depicted in blue. Values for samples from the Dilated Cardiomyopathy (DCM) cohort are orange. Significance values are given. Boxes with dashed lines indicate mRNA and with full lines metabolomics expression in blood (light blue shading) and left-ventricular heart tissue (pale red shading). RNAseq expression is given as (rlog-)normalized read counts. Metabolite concentrations are shown as $[\mu \mathrm{M}] . *: p<0.05 ;{ }^{*}: p<0.01$. ACO1 $=$ Aconitase $1 ; \mathrm{OGDH}=$ oxoglutarate dehydrogenase; DLST = dihydrolipoamide s-succinyltransferase. 


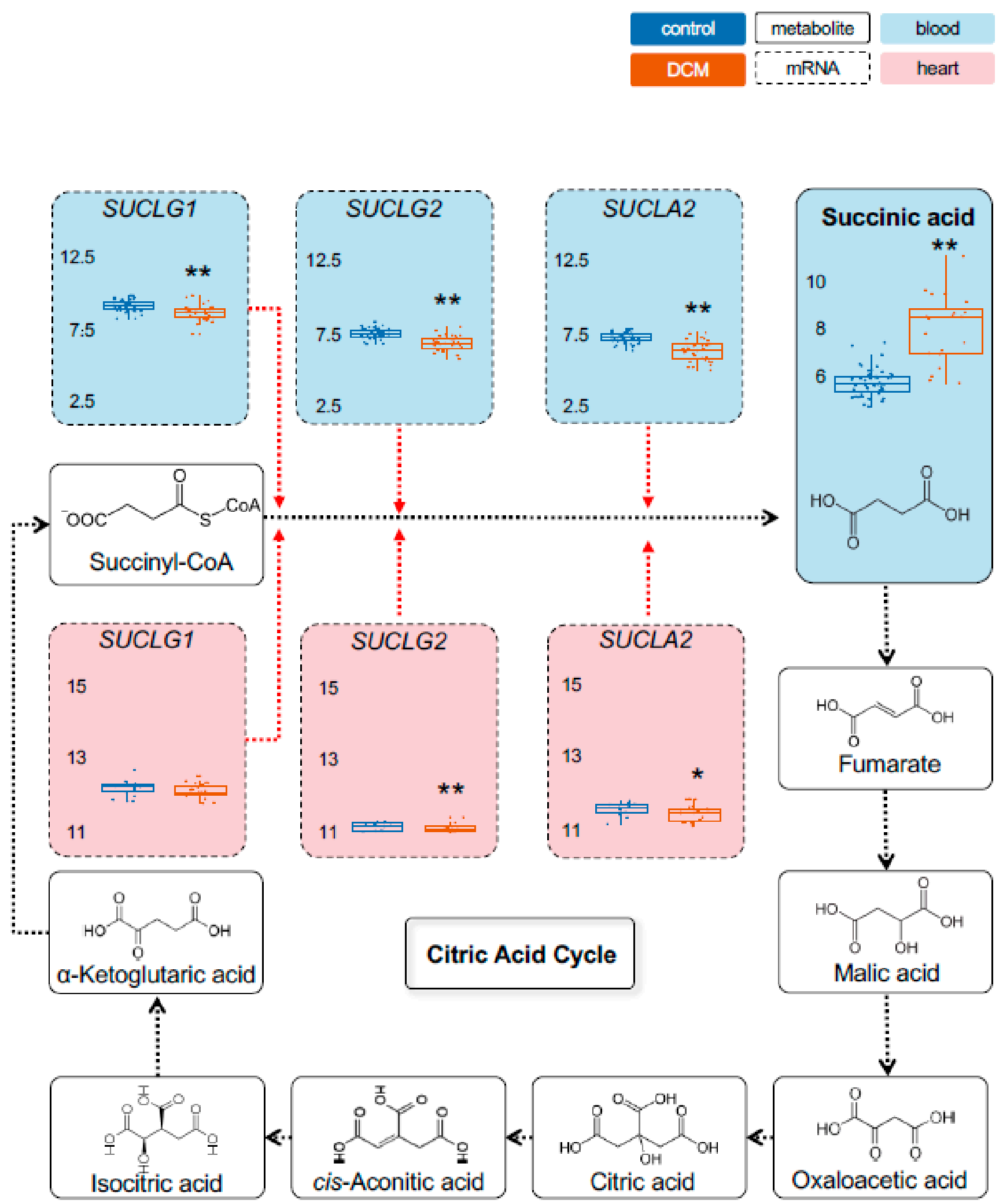

Figure 4. Detection of metabolic dysregulation in citric acid cycle. Following the citric acid cycle, succinic acid was Scheme 1. HF cohort, coinciding with decreased mRNA levels of several enzymes in blood. Same color and symbol annotation as in Figure 3. RNAseq expression is given as (rlog-)normalized read counts. Metabolite concentrations are shown as [ $\mu \mathrm{M}]$. *: $p<0.05 ;{ }^{* *}: p<0.01$. SUCLG1 = Succinate-CoA Ligase Alpha Subunit; SUCLG2 = succinate-coA ligase GDP-forming beta subunit; SUCLA2 = succinate-coA ligase ADP-forming beta subunit. 
control

A

\section{Pyruvic acid}

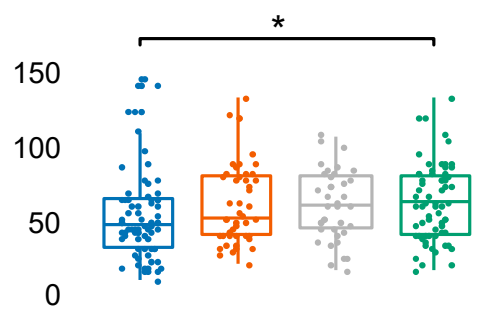

C

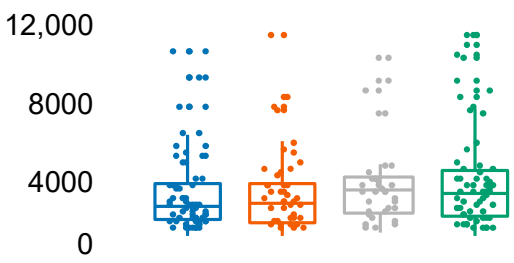

E

Isocitric acid

3

2

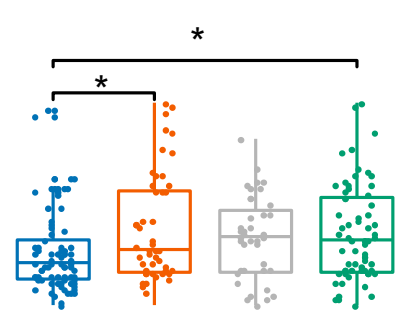

0

\section{DCM}

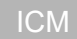

$\mathrm{DCM}+\mathrm{ICM}$

B

\section{a-Ketoglutaric acid}

40

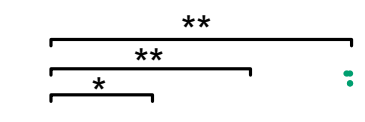

20

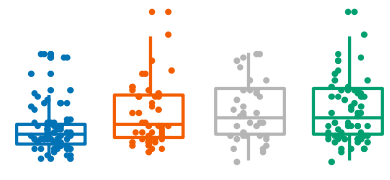

0

D

\section{Succinic acid}

10

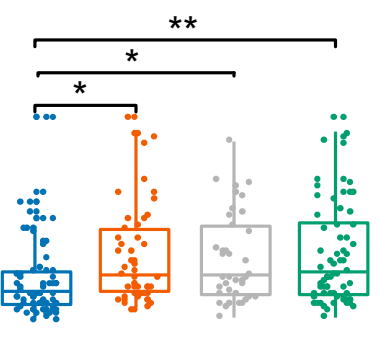

$\mathbf{F}$

Malic acid

40

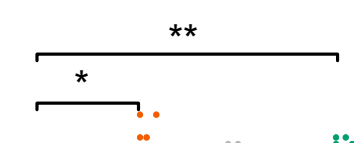

20

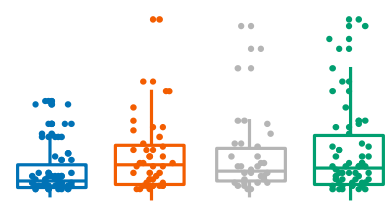

0

H

2-Hydroxybutyric acid

200

100

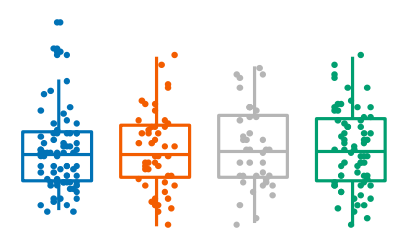

Figure 5. Validation in independent HF cohorts. While changes in pyruvic acid levels could not been replicated (A), the HF-related upregulation of $\alpha$-Ketoglutaric acid (B) was validated, while lactic acid (C) levels are not significantly altered in this cohort, in contrast to succinic acid (D), which was successfully validated. Isocitric and malic acid were also increased (E,F). Levels for 2-Hydroxybutyric and 2-Hydroxybutyric acid were found to be unaltered (G,H). Control (CTRL) samples are depicted in blue. Values for samples from the Dilated Cardiomyopathy (DCM) cohort are colored orange. Ischemic cardiomyopathy subgroup (ICM) is painted grey. Values for the combined cohort of DCM and ICM samples (DCM/ICM) are shown in green. Significance values are given. Metabolite concentrations are shown as $[\mu \mathrm{M}] .^{*}: p<0.05 ;{ }^{* *}: p<0.01$. 
The metabolic markers also included pyruvic acid (+oxaloacetic acid), which displayed a tendency to be elevated in DCM and ICM patients but did not reach statistical significance in the subgroups. However, the combined HF cohort showed significantly elevated pyruvic acid levels (1.5-fold, $p=0.032$ ) (Figure 5A). For lactic acid, our measurements showed a trend of higher levels in DCM as observed in the screening cohort but did not reach significance (Figure 5C). Additional metabolite information could be generated to fine map the energy metabolism changes. For instance, higher levels of isocitric acid were detected in DCM $(p=0.012)$ and in ICM $(p=0.067)(E)$. As shown in Figure 3 , the rate-limiting enzyme aconitase 1 (ACO1) was also significantly dysregulated in blood and heart in the multi-omics cohorts. Similarly, malic acid was increased in HF (DCM $p=0.019$; ICM $p=0.07$ ) (Figure 5F). For 3-hydroxybutyric acid (Figure 5G), DCM and ICM patients had a trend to lower levels compared to controls. No difference was seen for 2-hydroxybutyric acid (Figure 5H).

\section{Discussion}

The American Heart Association recently highlighted the potential for assessing metabolic states in cardiovascular diseases and made recommendations on future directions of human centered research in this area [7]. This is partly due to the fact that most seminal metabolic studies have been performed in vitro or in mice [8]. Although leading to important discoveries such as branched-chain amino acids (BCAA) accumulation [8], decreased cardiac fatty acid oxidation and increased ketone oxidation [9] and many other key-changes during HF and cardiac remodeling $[10,11]$, the studies resulted in relatively little understanding of the human system and its clinical relevance as well as the underlying regulatory principles in heart disease. To the best of our knowledge, this is the first multiOMICS study on energy metabolism in HF, combining transcriptomics and epigenomics, with quantitative metabolomics being the closest entity to the actual phenotype [12]. Since energetic changes are thought to be hallmarks of cardiac remodeling $[13,14]$, for example, by prolonged reliance on glucose utilization rather than fatty acid oxidation, we distinctly focused on energy metabolism in this study [10].

Lactate was one of the first biomarkers for HF and we can reproduce elevated levels only in part of our HF cohorts [2]. This might be due to the differences in disease severity (although LVEF was comparable) and underlines the limitations of this marker in daily clinical practice. Other findings seem to be more robust, such as the detection of increased levels of succinic acid. As Chouchani et al. showed in an ischemia reperfusion mouse model, the selective accumulation of the citric acid cycle intermediate succinate is a universal metabolic signature of ischemia in a range of tissues including the heart [15]. They pinpointed that increased succinate levels arise from reduced conversion to fumarate by the reversal of succinate dehydrogenase (SDH), when fumarate, aspartate and malate were available [15]. We here show significantly elevated succinate and malate levels in human systolic HF, with potential therapeutic implications. The described mechanism has also been validated by Zhang et al. [16]. However, they also proposed an accumulation of succinate in ischemia predominantly via the canonical Krebs-Cycle for example, through elevated $\alpha$-Ketoglutaric acid levels, which we also find in our study.

Interestingly, the metabolic changes also are reflected on the epigenetic and transcript level of several rate-limiting enzymes of this pathway, indicating that metabolic changes are at least partly driven by transcriptional regulation of the respective pathway.

Besides the biological validation in independent cohorts, we used gas chromatography mass spectrometry(GC-MS) instead of Liquid chromatography-mass spectrometry (LCMS/MS) for robust and reproducible independent metabolite separation and quantitation in the validation phase [10]. The applicability of both methods has been recently shown for example, by Mueller et al. in a study on metabolites in a cardiac hypertrophy and heart failure model [17]. Potential limitations of this study include its monocentric nature with potential referral bias of our tertiary center. To address this, the findings have been carefully validated in three independent cohorts of $\mathrm{HF}$, also including ischemic HF to show generalizability. Another potential limitation is the higher number of diabetic patients compared 
to controls, which could lead to a metabolic effect driven by insulin resistance [18], which is a known effect especially in diabetic and obese patients [19]. However, by adjusting our cohort to glucose (H1) levels (see M\&M and Supplemental Figure S1), we prevent such a potential effect, as $\mathrm{H} 1$ has been shown to be significantly different in type-2 diabetes compared to controls [20].

In summary, we have detected significant dysregulation related to energy production pathways in HF at different OMICs-levels, showing its importance in disease onset and progression and suggest a possible use of distinct molecules like succinic acid as an (early) biomarker and interventional target in HF.

\section{Materials and Methods}

\subsection{Patient Enrolment and Study Design}

Our study was approved by the ethics committee of Heidelberg University. All participants have given written informed consent to allow for molecular analysis of blood and left-over tissue for the identification of novel biomarkers (appl. no. S-390/2011). The diagnosis of Dilated Cardiomyopathy (DCM) was confirmed after excluding coronary artery disease as determined by coronary angiography, valvular heart disease was excluded by cardiac magnetic resonance imaging (cMRI) and echocardiography and myocarditis/inflammatory DCM by histopathology [21]. Patients routinely biopsied after heart transplantation served as controls for epigenetic and transcriptomic studies. Importantly, only patients with normal cardiac function and without transplant rejection were included as controls.

We performed a two-step approach and recruited first a screening cohort including $n=82 \mathrm{DCM}$ patients and $n=51$ controls and afterwards a second stage cohort of $n=52 \mathrm{DCM}$, $n=39$ ischemic cardiomyopathy (ICM) patients and $n=57$ controls. The ICM patients in the second stage had relevant coronary artery disease as judged by coronary angiography in combination with decreased systolic function. Clinical controls were recruited at the study side and they had a cardiovascular risk profile but neither HF or relevant coronary artery disease, as judged by a recent coronary angiography.

\subsection{Biomaterial Processing}

For all subjects, blood draw was performed without requiring a special fasting protocol. For serum samples, an $8 \mathrm{~mL}$ aliquot of blood samples from each participant was collected directly into serum collection tubes (Sarstedt Monovette). The serum samples were allowed to stand for $1 \mathrm{~h}$ at room temperature before being centrifuged at $3000 \mathrm{rpm}$ for $10 \mathrm{~min}$. Then, the serum supernatant was recovered and stored at $-80^{\circ} \mathrm{C}$ until further analysis. In total $200 \mu \mathrm{L}$ serum were sent to Biocrates (Innsbruck) on dry ice, where metabolomic profiling was performed. Biopsies for transcriptome analysis have been handled as described in [22].

\subsection{Metabolomics Profiling}

In the screening cohort the AbsoluteIDQ p180 kit, as well as Specialized assay kits including assays for Amino acids \& biogenic amines, Acylcarnitines, Lysophophosphatidylcholine, Phosphatidylcholine, Sphingomyelins, Hexoses, Prostaglandins, Bile acids and the Energy metabolism (Biocrates Life Sciences AG, Innsbruck, Austria) were used to quantify in total 435 metabolites, of which 381 had detection levels meeting quality standards. Hexoses were measured using a fully automated assay based on PITC (phenylisothiocyanate)derivatization in the presence of internal standards followed by flow injection analysis (FIA)- tandem mass spectrometry (MS/MS) using an AB SCIEX 4000 QTrap ${ }^{\circledR}$ mass spectrometer (AB SCIEX, Darmstadt, Germany) with electrospray ionization. The experimental metabolomics measurement technique is described in detail by patent US 2007/0004044 accessible online at http:/ / www.freepatentsonline.com/20070004044.html, last accessed 15 February 2021). For the quantitative analysis of energy metabolism intermediates involved in glycolysis, citrate cycle or pentose phosphate pathway, urea cycle hydrophilic interaction liquid chromatography (HILIC)-Electrospray ionization ESI-MS/MS 
was performed using a SCIEX 4000 QTrap $^{\mathrm{TM}}$ tandem mass spectrometer (Applied Biosystems/MDS Analytical Technologies) for multiple reaction monitoring (MRM). Protein was precipitated and extracted simultaneously from samples with methanol in a 96 well plate format. Ten trade secret internal standards (ratio of external to internal standard) and external calibration were used for highly accurate metabolomics quantitation, including lactate (Lac), alpha-ketoglutaric acid (alpha-KGA), succinic acid (Suc) and pyruvic acid (+oxaloacetic acid) (Pyr\&OAA).

Metabolites of the energy metabolism of samples from the second stage cohorts were measured at a later timepoint according to the following procedure: After derivatization to their corresponding methoxime-trimethylsilyl (MeOx-TMS) derivatives, energy metabolites were determined by gas chromatography-mass spectrometry (GC-MS) using Agilent 7890 GC/5975 MSD (Agilent, Santa Clara, CA, USA) instruments. Pretreated samples were evaporated until completely dry and subjected to a two-step methoximation-silylation derivatization. $\mathrm{N}$-methyl- $\mathrm{N}$-(trimethylsilyl) trifluoroacetamide (MSTFA) was used as sialylation reagent. Split injection was performed and chromatograms were recorded in selected ion monitoring (SIM) mode. External standard calibration curves and ten internal standards were used to calculate concentrations of individual energy metabolites. Data were quantified using the appropriate mass spectrometry software (Agilent MassHunter) and imported into the Biocrates MetIDQTM software for further analysis.

As it is known that glycolysis can proceed in sampling tubes in a time depended manner, resulting in a decrease of glucose coupled with an increase of lactate [23,24], we excluded DCM samples from the analysis with H1-(Hexoses including glucose) levels less than 2-fold times standard deviation from the mean control H1-levels. H1-levels of the harmonized cohorts are shown in Supplemental Figure S1.

\subsection{Transcriptome and Epigenome Analysis}

RNAseq-analysis for polyA-enriched mRNA and measurements of CpG sites by illumina $450 \mathrm{k}$ methylation array have been performed as described earlier [22]. In detail, available samples were sequenced to a median paired-end read count of 29.85 million. Unstranded paired-end raw read files were mapped with STAR [25] (v2.4.1c5) using GRCh37/hg19 and the Gencode 19 gene model (http:/ / www.gencodegenes.org/, accessed on 17 February 2021). Only uniquely mapped reads were counted into genes using subread's feature counts program [26] (subread version 1.4.6.p1) and mapping percentages were median 88.08. Count data were normalized by rlog normalization [27].

\subsection{Statistical Analysis}

To account for outliers in the raw metabolomics and methylation and RNA data, we performed outlier detection using Rosner's Test for Outliers with from the EnvStats(v.2.3.1) package available for R through Bioconductor (https: / / www.rdocumentation.org/ packages/EnvStats, accessed on 17 February 2021), with $k=4$ indicating the number of suspected outliers. After removal of outliers, $p$-values were calculated with help of the t.test function from the "stat_compare_means" function of the R-package ggpubr- (v.0.2.999) (https://www.rdocumentation.org/packages/ggpubr, accessed on 17 February 2021). $p$-values for individual CpG-sites have been aggregated by gene using Simes's method for combining $p$-values (https://rdrrio/cran/mppa/man/simes.test.html, accessed on 17 February 2021) [28].

Supplementary Materials: The following are available online at https:/ /www.mdpi.com/1422-0 067/22/4/1999/s1, Figure S1: H1 metabolite levels (screening); Figure S2: Principle component analysis of metabolites (screening); Figure S3: Detection of Metabolites and associated enzyme mRNA expression (screening); Figure S4: Validation of cardiac gene expression patterns; Figure S5: Detection of Metabolites and associated enzyme methylation (screening).

Author Contributions: Conceptualization, B.M.; methodology, B.M. and J.H.; software, J.H.; validation, J.H., B.M.; formal analysis, J.H.; investigation, J.H., K.S.F., F.S.-H., E.K., R.T., R.N., O.F.T., M.W., 
A.A.; resources, C.D., T.W., T.N.; writing-original draft preparation, B.M., J.H.; writing-review and editing, M.P.M., T.K., M.D., U.V., J.F., N.F., S.B.F., A.K., H.A.K.; visualization, J.H.; supervision, B.M.; project administration, B.M.; funding acquisition, B.M. All authors have read and agreed to the published version of the manuscript.

Funding: This work was partially supported by grants from the DZHK ("Deutsches Zentrum für Herz-Kreislauf-Forschung"-German Centre for Cardiovascular Research), Detectin-HF (ERA-CVD) and Siemens Healthcare GmbH (Siemens/University Heidelberg Joint Research Project: Care4DCM). Bioinformatics and multi-omics data analysis was supported by informatics for life.

Institutional Review Board Statement: The study was conducted according to the guidelines of the Declaration of Helsinki. Biobanking and biomarker analysis was approved by the ethics committee of Heidelberg University (appl. no. S-390/2011).

Informed Consent Statement: Informed consent was obtained from all subjects involved in the study, allowing molecular analysis of blood and left-over tissue for the identification of novel biomarkers.

Data Availability Statement: Aggregated data of DNA methylation arrays and mRNA sequencing of the case and controls cohorts are available at https:/ / ccb-web.cs.uni-saarland.de/cms, accessed on 17 February 2021.

Conflicts of Interest: C.D. and T.N. are employees of Siemens Healthcare GmbH. B.M. and J.H. hold patent applications for biomarkers.

\section{References}

1. Bertero, E.; Maack, C. Metabolic remodelling in heart failure. Nat. Rev. Cardiol. 2018, 15, 457-470. [CrossRef] [PubMed]

2. Ritterhoff, J.; Tian, R. Metabolism in cardiomyopathy: Every substrate matters. Cardiovasc. Res. 2017, 113, 411-421. [CrossRef]

3. Coronel, R.; De Groot, J.R.; Van Lieshout, J.J. Defining heart failure. Cardiovasc. Res. 2001, 50, 419-422. [CrossRef]

4. Tan, L.-B.; Williams, S.G.; Tan, D.K.H.; Cohen-Solal, A. So many definitions of heart failure: Are they all universally valid? A critical appraisal. Expert Rev. Cardiovasc. Ther. 2010, 8, 217-228. [CrossRef]

5. Savarese, G.; Lund, L.H. Global Public Health Burden of Heart Failure. Card. Fail. Rev. 2017, 3, 7-11. [CrossRef]

6. Neubauer, S. The failing heart-An engine out of fuel. N. Engl. J. Med. 2007, 356, 1140-1151. [CrossRef]

7. Cheng, S.; Shah, S.H.; Corwin, E.J.; Fiehn, O.; Fitzgerald, R.L.; Gerszten, R.E.; Illig, T.; Rhee, E.P.; Srinivas, P.R.; Wang, T.J.; et al. Potential Impact and Study Considerations of Metabolomics in Cardiovascular Health and Disease: A Scientific Statement from the American Heart Association. Circ. Cardiovasc. Genet. 2017, 10, e000032. [CrossRef]

8. Sansbury, B.E.; De Martino, A.M.; Xie, Z.; Brooks, A.C.; Brainard, R.E.; Watson, L.J.; DeFilippis, A.P.; Cummins, T.D.; Harbeson, M.A.; Brittian, K.R.; et al. Metabolomic analysis of pressure-overloaded and infarcted mouse hearts. Circ. Heart Fail. 2014, 7, 634-642. [CrossRef]

9. Aubert, G.; Martin, O.J.; Horton, J.L.; Lai, L.; Vega, R.B.; Leone, T.C.; Koves, T.; Gardell, S.J.; Krüger, M.; Hoppel, C.L.; et al. The Failing Heart Relies on Ketone Bodies as a Fuel. Circulation 2016, 133, 698-705. [CrossRef]

10. McGarrah, R.W.; Crown, S.B.; Zhang, G.-F.; Shah, S.H.; Newgard, C.B. Cardiovascular Metabolomics. Circ. Res. 2018, 122, 1238-1258. [CrossRef]

11. Torella, D.; Ellison, G.M.; Torella, M.; Vicinanza, C.; Aquila, I.; Iaconetti, C.; Scalise, M.; Marino, F.; Henning, B.J.; Lewis, F.C.; et al. Carbonic Anhydrase Activation Is Associated with Worsened Pathological Remodeling in Human Ischemic Diabetic Cardiomyopathy. J. Am. Heart Assoc. 2014, 3, e000434. [CrossRef] [PubMed]

12. Johnson, C.H.; Ivanisevic, J.; Siuzdak, G. Metabolomics: Beyond biomarkers and towards mechanisms. Nat. Rev. Mol. Cell Biol. 2016, 17, 451-459. [CrossRef] [PubMed]

13. Azevedo, P.S.; Minicucci, M.F.; Santos, P.P.; Paiva, S.A.R.; Zornoff, L.A.M. Energy Metabolism in Cardiac Remodeling and Heart Failure. Cardiol. Rev. 2013, 21, 135-140. [CrossRef]

14. Ingwall, J.S. Energy metabolism in heart failure and remodelling. Cardiovasc. Res. 2009, 81, 412-419. [CrossRef]

15. Chouchani, E.T.; Pell, V.R.; Gaude, E.; Aksentijević, D.; Sundier, S.Y.; Robb, E.L.; Logan, A.; Nadtochiy, S.M.; Ord, E.N.; Smith, A.C.; et al. Ischaemic accumulation of succinate controls reperfusion injury through mitochondrial ROS. Nature 2014, 515, 431-435. [CrossRef]

16. Zhang, J.; Wang, Y.T.; Miller, J.H.; Day, M.M.; Munger, J.C.; Brookes, P.S. Accumulation of Succinate in Cardiac Ischemia Primarily Occurs via Canonical Krebs Cycle Activity. Cell Rep. 2018, 23, 2617-2628. [CrossRef]

17. Muller, O.J.; Heckmann, M.B.; Ding, L.; Rapti, K.; Rangrez, A.Y.; Gerken, T.; Christiansen, N.; Rennefahrt, U.E.E.; Witt, H.; Maldonado, S.G.; et al. Comprehensive plasma and tissue profiling reveals systemic metabolic alterations in cardiac hypertrophy and failure. Cardiovasc. Res. 2019, 115, 1296-1305. [CrossRef]

18. Abel, E.D.; O'Shea, K.M.; Ramasamy, R. Insulin resistance: Metabolic mechanisms and consequences in the heart. Arterioscler. Thromb. Vasc. Biol. 2012, 32, 2068-2076. [CrossRef] [PubMed] 
19. Sasso, F.C.; Carbonara, O.; Nasti, R.; Marfella, R.; Esposito, K.; Rambaldi, P.; Mansi, L.; Salvatore, T.; Torella, R.; Cozzolino, D. Effects of insulin on left ventricular function during dynamic exercise in overweight and obese subjects. Eur. Heart J. 2005, 26, 1205-1212. [CrossRef]

20. Knebel, B.; Strassburger, K.; Szendroedi, J.; Kotzka, J.; Scheer, M.; Nowotny, B.; Mussig, K.; Lehr, S.; Pacini, G.; Finner, H.; et al. Specific Metabolic Profiles and Their Relationship to Insulin Resistance in Recent-Onset Type 1 and Type 2 Diabetes. J. Clin. Endocrinol. Metab. 2016, 101, 2130-2140. [CrossRef] [PubMed]

21. Richardson, P.; McKenna, W.; Bristow, M.; Maisch, B.; Mautner, B.; O'Connell, J.; Olsen, E.; Thiene, G.; Goodwin, J.; Gyarfas, I.; et al. Report of the 1995 World Health Organization/International Society and Federation of Cardiology Task Force on the Definition and Classification of cardiomyopathies. Circulation 1996, 93, 841-842. [PubMed]

22. Meder, B.; Haas, J.; Sedaghat-Hamedani, F.; Kayvanpour, E.; Frese, K.; Lai, A.; Nietsch, R.; Scheiner, C.; Mester, S.; Bordalo, D.M.; et al. Epigenome-Wide Association Study Identifies Cardiac Gene Patterning and a Novel Class of Biomarkers for Heart Failure. Circulation 2017, 136, 1528-1544. [CrossRef]

23. Astles, R.; Williams, C.P.; Sedor, F. Stability of plasma lactate in vitro in the presence of antiglycolytic agents. Clin. Chem. 1994, 40 Pt 1, 1327-1330. [CrossRef]

24. Oddoze, C.; Lombard, E.; Portugal, H. Stability study of 81 analytes in human whole blood, in serum and in plasma. Clin. Biochem. 2012, 45, 464-469. [CrossRef]

25. Dobin, A.; Davis, C.A.; Schlesinger, F.; Drenkow, J.; Zaleski, C.; Jha, S.; Batut, P.; Chaisson, M.; Gingeras, T.R. STAR: Ultrafast universal RNA-seq aligner. Bioinformatics 2013, 29, 15-21. [CrossRef] [PubMed]

26. Liao, Y.; Smyth, G.K.; Shi, W. featureCounts: An efficient general purpose program for assigning sequence reads to genomic features. Bioinformatics 2014, 30, 923-930. [CrossRef]

27. Love, M.I.; Huber, W.; Anders, S. Moderated estimation of fold change and dispersion for RNA-seq data with DESeq2. Genome Biol. 2014, 15, 550. [CrossRef]

28. Simes, R.J. An improved Bonferroni procedure for multiple tests of significance. Biometrika 1986, 73, 751-754. [CrossRef] 